\title{
Cerebellar Ataxia with Progressive Optic Atrophy and Deafness (CAPOS Syndrome): A Rare Case Report
}

\author{
MUZAHID MAA ${ }^{1}$, CHOWDHURY $^{2}$, SAHA ${ }^{3}$, ROY $U^{4}$, KABIR MS $^{5}$, SARKER I ${ }^{6}$, ISLAM MR ${ }^{6}$
}

\begin{abstract}
CAPOS syndrome (cerebellar ataxia, areflexia, pes cavus, optic atrophy, sensorineural hearing loss) is a rare autosomal dominant disorder caused by ATP1A3 mutation. Fever triggered cerebellar dysfunction along with progressive optic atrophy and sensorineural deafness are typical features. Herein, we report a 20-year-old female with multiple fever induced cerebellar dysfunction with partial improvement. Pendular nystagmus and optic atrophy were prominent which were also present in patient's younger sister. As there was progressive sensorineural hearing loss and areflexia, CAPOS syndrome was diagnosed. Though specific therapy is yet to be developed, acetazolamide, cochlear implantation, visual aid and family screening may improve quality of life.
\end{abstract}

Key words: CAPOS syndrome, cerebellar ataxia, optic atrophy, hearing loss, ATP1A3 mutation.

Introduction:

CAPOS syndrome, which is derived from the acronym of symptoms like cerebellar ataxia, areflexia, pes cavus, optic atrophy, sensory neural hearing loss, is a very rare disorder; first described in three patients by Nicolaides et al. in $1996^{1}$. Since the original description, about forty patients have been reported and causal association with specific genetic mutation has been established ${ }^{2}$. A missense mutation of the ATP1A3 gene, inherited as autosomal dominant traits with variable expressivity, causes the allelic disorders namely: CAPOS syndrome, rapid - onset dystoniaparkinsonism (RDP) in DYT12 and alternating hemiplegia of childhood $(\mathrm{AHC})^{3}$. The typical CAPOS phenotype presents in infancy to early childhood with recurrent episodes of neurological dysfunction usually triggered by non-specific febrile illness ${ }^{4}$. Usually there is one to three paroxysmal episodes of cerebellar ataxia, areflexia, hypotonia, motor weakness, ophthalmoparesis, lethargy and/ or comatose state that resolve within days to weeks, leaving residual ataxia, areflexia as the deafness and optic atrophy gradually deteriorate ${ }^{5,6}$. Less-well reported features include seizures, choreo-athetosis, dystonia, autistic features, mild learning disabilities which may suggest partial overlap of other ATP1A3-related disorders (RDP \& AHC) with CAPOS phenotype ${ }^{7}$. But it is the progressive optic atrophy and sensorineural deafness along with cerebellar ataxia that differentiates CAPOS phenotype from others ${ }^{7,8}$.

Here, we report the case of a CAPOS syndrome with typical presentation, who also has a sibling showing early features of the same syndrome.

\section{Case Report:}

The patient is a 20-year-old Bangladeshi female, who is the first issue of healthy consanguineous parents. She was born at term after an uneventful pregnancy by normal vaginal delivery. Her birth weight was more than 2500 gram with no features of perinatal asphyxia. Her growth and development were age appropriate up to 8 months of age, after which she developed non-specific fever for 2 days.

1. Dr. Md. Abdullah Al Muzahid, Phase B Resident, Dept of Neurology, BSMMU, Dhaka.

2. Dr. Ashish Chowdhury, Phase B Resident, Dept of Neurology, BSMMU, Dhaka.

3. Dr. Sujan Saha, Phase B Resident, Dept of Neurology, BSMMU, Dhaka.

4. Dr. Uttam Roy, Phase B Resident, Dept of Neurology, BSMMU, Dhaka.

5. Dr. Md. Suman Kabir, Phase B Resident, Dept of Neurology, BSMMU, Dhaka.

6. Dr. Imran Sarker, Assistant Professor (Clinical Neurology), NINS\&H, Dhaka

7. Dr. Md. Rafiqul Islam, Professor And Chairman, Dept of Neurology, BSMMU, Dhaka. 
During the febrile episode, she showed irritability, lethargy, hypotonia and pendular movement of both eyes. She also showed occasional sudden jerky movement of hands and feet, 2-3 times per day. She was hospitalized and treated with intra-venous medications and fluid and responded within days. As her condition improved, she showed persistent pendular movement of both eyes with difficulty in eye fixation. She had mild developmental delay showing clumsiness in standing and walking. But she could walk independently at 2.5 years.

At about 10 years of age, she developed fever for 3-4 days with decreased muscle power, diminished responsiveness and inability to walk. But within few days her condition improved as she could walk independently again. But her parents noticed, her speech became slurred with some word becoming more difficult to pronounce. Her eyes became shakier; she showed more difficulty on walking on uneven surfaces. She had difficulty fixating her eyes to text and also hearing faint sound became problematic for her. As a consequence her school performance became poorer. They consulted ophthalmologist who prescribed spectacles and advised reading in well illuminated conditions. But gradually her hearing and vision diminished. Initially she had corrected vision of $6 / 6$ with spectacles. But within years her best corrected vision became $6 / 36$ bilaterally. As for her hearing, audiometry initially showed mild sensorineural deafness that progressively became moderately severe bilaterally. She also developed occasional myoclonic jerking involving part of body for which she was evaluated by a neurologist who advised electro-encephalogram and prescribed antiepileptic drug with which her jerking improved.

She stopped school at 16 years of age at class nine due to poor vision and hearing. Her age of menarche was at 13 years. Her menstrual cycle was regular with average flow. She was unmarried. She was immunized according to national immunization schedule. All her family members except for her younger sister were in good health. There was no history of such illness in her family members.

At time of presentation to us, she was alert with body built below average. Vitals were within normal limit with no anemia, jaundice, clubbing, thyromegaly, lymph node enlargement. She had no abnormal skin pigmentation, high arched palate, scoliosis, pes cavus or any chest deformity. Nervous system examination revealed, she was oriented in time, place and person, memory was normal, intelligence below average, and speech was slurred with inability to pronounce properly. She occasionally talked to herself, but there was no abnormal thought or any abnormal neologism. Regarding cranial nerve examination, her eye had full range of motion with pendular horizontal nystagmus that showed no fatigue or latency. Fundoscopy revealed bilateral primary optic atrophy and Rinne-Weber test revealed bilateral sensorineural hearing loss. All other cranial nerves were intact. Motor system revealed normal muscle bulk with hypotonia, power of all 4 limbs were MRC grade5. All jerks were diminished to absent with re-inforcement and planter were bilaterally flexor. She had no abnormal movement of any part of body at rest. Her coordination was impaired as evidenced by dysmetria, dysdiadochokinesia and abnormal heel-shin test. Her gait was broad based with irregular steps and her tandem gait was impaired. She had difficulty walking on uneven surfaces and going up/ downstairs. All modalities of sensation including vibration and position sense were intact. She could do her activities of daily life without any support.

She underwent extensive investigation and consulted different physicians of different specialties. List of her investigation profile is given below. But except for optic atrophy and sensori-neural hearing loss, all her investigations revealed no clue to the cause of her abnormality. The only investigation that could have been done to establish the etiology was genetic analysis, but patient party refused to proceed further for lack of financial support.

Patient's younger sister, 8 years of age also had pendular movement of both eyes along with impairment of tandem gait, triggered by febrile illness. She had no other cerebellar feature. She also had mild visual difficulties and optic disc pallor. Her hearing was normal at bedside testing. She had no pes cavus. The patient's younger brother was quite normal. 


\section{Laboratory investigations done on the patient}

Blood

Urine

CSF

Imaging

Complete blood count, ESR, CRP, glucose, urea, creatinine, electrolytes, liver function, thyroid hormone, iron profile, creatine kinase, Antinuclear and antipospholipid antibody, ANCA, VDRL, hepatitis B\&C antibody, ceruloplasmin, vitamin B12, folate

Routine examination, culture sensitivity, copper

Cytology, protein, glucose, VDRL, ADA, lactate, Gram \& AFB stain, Gene xpert for MTB, oligo-clonal band \& lg-G index

Chest Xray, Ultrasonogram of whole abdomen, ECG, Echocardiogram, MRI of brain and orbit, slit lamp examination for KF ring

Neuro-physiology Electro-encephalogram, nerve conduction study

\section{Discussion:}

Cerebellar ataxia may impose a diagnostic dilemma as there are many differentials, but the age of onset, speed of progression, family history and concomitant other features may provide clue to the final diagnosis ${ }^{5}$. CAPOS syndrome is a rare differential of cerebellar syndrome. Though first described in 1996, it remains a rare condition and the underlying pathogenesis is yet to be fully described ${ }^{4}$.

This is the first case report of CAPOS syndrome from Bangladesh, to the best of our knowledge. Among the core features, our patient did not have pes cavus. All the case reports that have been published so far, only $30 \%$ of them had pes cavus ${ }^{9}$. As a matter of fact, some authors also suggested changing the acronym to "CAOS" syndrome; as all the patients did not have pes cavus ${ }^{6}$. Our patient presented with the typical history of more than one fever induced cerebellar ataxia with encephalopathic features and weakness with partial remission. But her nystagmus and areflexia persisted as the vision and hearing gradually deteriorated, which is consistent with typical presentation ${ }^{7,10}$. She also had delayed milestone of development, mild intellectual impairment and few episodes of seizure which are among less-well reported features; but are consistent with spectrum of ATP1A3 mutation phenotype ${ }^{11}$. The patient and her younger sister were affected among three siblings, which may indicate the autosomal dominant pattern of inheritance. But as the parents were not affected, it might be the de novo mutation that may explain the disease, which is also well reported ${ }^{5}$.

CAPOS syndrome is caused by missense mutation of the ATP1A3 gene with replacement of $818^{\text {th }}$ amino acid glutamic acid by lysine ${ }^{5,6}$. Other diseases RDP, AHC and most recently relapsing encephalopathy with cerebellar ataxia have also been attributed to the ATP1A3 mutation ${ }^{12}$. These disorders have overlapping features like early onset, triggered by febrile illness, polyphasic episodes with abnormal movements of different parts of body including eyes, seizure potentials and occasional intellectual disabilities ${ }^{6}$. But the differentiating features of CAPOS phenotype are the progressive bilateral optic atrophy and sensorineural deafness ${ }^{6}$.

During the acute episodes clinical features may be compatible with encephalitis or post viral cerebellitis; or if motor weakness is pronounced then atypical $\mathrm{GBS}^{9}$. Also due to the relapsing and remitting course, multiple sclerosis may also be considered. But the age of onset and absence of relevant imaging and CSF findings excluded MS. Again Friedreich's ataxia was also a differential but presence of hearing loss and absence of sensory neuropathy and abnormal imaging excluded this consideration.

ATP1A3 encodes for alpha 3 subunit of the $\mathrm{Na}^{+} /$ $\mathrm{K}^{+}$-ATPase pump which is a ubiquitous transmembrane enzyme responsible for 
maintaining cell membrane polarity ${ }^{12}$. The alpha 3 subunit is selectively present in the neurons of CNS, whereas alpha 2 subunit is expressed in the glial cells ${ }^{10}$. Alpha 3 subunit is also expressed in the afferent and efferent neurons innervating the skeletal muscle spindle which may explain the acquired areflexia without any electro-physiological evidence of peripheral neuropathy ${ }^{6}$. The sensorineural hearing loss is attributed to auditory neuropathy and best treated with cochlear implantation as it directly stimulates the neural pathway ${ }^{7}$. Optic atrophy adds more obstacle in the way of communication as deaf persons often rely on visual cues for lip reading.

Till date, there is no established disease modifying therapy for CAPOS syndrome $7,9,10$. Carbonic anhydrase inhibitor acetazolamide has been reported to prevent relapse by generating a state of metabolic and brain acidosis, which may normalize neuronal excitability ${ }^{5,9}$. Supportive treatment with visual aid, cochlear implantation, speech therapy may improve quality of life to some extent ${ }^{7}$.

\section{Conclusion:}

CAPOS syndrome is an autosomal dominant disorder presenting as cerebellar ataxia with progressive optic atrophy and sensori-neural deafness. When there is no imaging and electrophysiological evidence of any abnormality; CAPOS syndrome remains an important differential. Genetic analysis to see ATP1A3 mutation establishes the diagnosis. Supportive therapy along with acetazolamide may be of benefit. But counseling and screening of other family members are equally important.

\section{Conflict of interests:}

The authors declare that they have no conflict of interest.

\section{References:}

1. Nicolaides P, Appleton RE, Fryer A. Cerebellar ataxia, areflexia, pes cavus, optic atrophy, and sensorineural hearing loss (CAPOS): a new syndrome. Journal of medical genetics. 1996 May 1;33(5):419-21.
2. Stenshorne I, Rasmussen $M$, Salvanos $P$, Tallaksen CM, Bindoff LA, Koht J. Feverrelated ataxia: a case report of CAPOS syndrome. Cerebellum \& ataxias. 2019 Dec 1;6(1):2.

3. Demos MK, van Karnebeek CD, Ross CJ, Adam S, Shen Y, Zhan SH, Shyr C, Horvath G, Suri M, Fryer A, Jones SJ. A novel recurrent mutation in ATP1A3 causes CAPOS syndrome. Orphanet journal of rare diseases. 2014 Dec 1;9(1):15.

4. Paquay S, Wiame E, Deggouj N, Boschi A, De Siati RD, Sznajer Y, Nassogne MC. Childhood hearing loss is a key feature of CAPOS syndrome: A case report. International Journal of Pediatric Otorhinolaryngology. 2018 Jan 1;104:191-4.

5. Maas RP, Schieving JH, Schouten M, Kamsteeg EJ, van de Warrenburg BP. The genetic homogeneity of CAPOS syndrome: four new patients with the c. 2452G>A (p. Glu818Lys) mutation in the ATP1A3 gene. Pediatric neurology. 2016 Jun 1;59:71-5.

6. Heimer G, Sadaka Y, Israelian L, Feiglin A, Ruggieri A, Marshall CR, Scherer SW, Ganelin-Cohen E, Marek-Yagel D, Tzadok M, Nissenkorn A. CAOS-Episodic cerebellar ataxia, areflexia, optic atrophy, and sensorineural hearing loss: A third allelic disorder of the ATP1A3 gene. Journal of Child Neurology. 2015 Nov;30(13):1749-56.

7. Tranebjærg L, Strenzke N, Lindholm S, Rendtorff ND, Poulsen H, Khandelia H, Kopec W, Lyngbye TJ, Hamel C, Delettre C, Bocquet B. The CAPOS mutation in ATP1A3 alters $\mathrm{Na} /$ K-ATPase function and results in auditory neuropathy which has implications for management. Human genetics. 2018 Feb 1;137(2):111-27.

8. Hayashida T, Saito Y, Ishii A, Hirose S, Hiraiwa $\mathrm{R}$, Maegaki $\mathrm{Y}$, Ohno K. Further characterization of CAPOS/CAOS syndrome with the Glu818Lys mutation in the ATP1A3 gene: a case report. Brain and Development. 2018 Aug 1;40(7):576-81. 
9. Rodriguez AD, Prochazkova M, Santos SS, Cabezas OR, Extremera VC, GonzalezGutierrez-Solana L. Early Diagnosis of CAPOS Syndrome Before Acute-Onset Ataxia-Review of the Literature and a New Family. Pediatric Neurology. 2017 Jun 1;71:60-4.

10. Carecchio M, Zorzi G, Ragona F, Zibordi F, Nardocci N. ATP1A3-related disorders: an update. European Journal of Paediatric Neurology. 2018 Mar 1;22(2):257-63.
11. Sweney MT, Newcomb TM, Swoboda KJ. The expanding spectrum of neurological phenotypes in children with ATP1A3 mutations, Alternating Hemiplegia of Childhood, Rapid-onset DystoniaParkinsonism, CAPOS and beyond. Pediatric neurology. 2015 Jan 1;52(1):56-64.

12. Dard R, Mignot $C$, Durr A, Lesca G, Sanlaville $D$, Roze E, Mochel F. Relapsing encephalopathy with cerebellar ataxia related to an ATP 1A3 mutation. Developmental Medicine \& Child Neurology. 2015 Dec;57(12):1183-6. 